passes during MT were independently associated with DHC in anterior circulation EVLO patients achieving SR at the end of MT. Of these, admission glucose levels and number of passes during MT are potentially modifiable factors.

Disclosures N. Goyal: None. G. Tsivgoulis: None. A. Pandhi: None. M. Ishfaq: None. J. Goyanes: None. A. Deep: None. D. Alsbrook: None. S. Singh: None. G. Zaid: None. D. Hoit: None. C. Nickele: None. V. Inoa: None. D. Dornbos: None. A. Alexandrov: None. A. Arthur: None. L. Elijovich: None.

\section{E-108 LONG TERM CLINICAL AND ANGIOGRAPHIC OUTCOME WITH THE INTRACRANIAL STENT FOR SYMPTOMATIC INTRACRANIAL STENOSIS}

'S Park*, ${ }^{2} \mathrm{~S}$ Seo, 'J Kim. 'Neurosurgery, Gangnam Severance Hospital, Yonsei University, Seoul, Korea, Republic of; ${ }^{2}$ Neuroradiology, Gangnam Severance Hospital, Yonsei University, Seoul, Korea, Republic of

\subsection{6/neurintsurg-2020-SNIS. 141}

Background and Aims The risk of ischemic stroke from a symptomatic stenotic intracranial artery is high despite best medical therapy (BMT). Clinicians have increasingly turned to percutaneous transluminal angioplasty and stenting (PTAS) over the last decades as an alternative therapy in high-risk patient with symptomatic ICAS. We evaluated long-term clinical outcome and restenosis rate with atherosclerotic intracranial stenosis using the Wingspan self-expanding nitinol stent system.

Methods The patients treated with the Wingspan in two medical center from January 2010 to December 2016 were enrolled. Target patients were affected by high-grade, symptomatic, intracranial atherosclerotic lesions, were on antithrombotic therapy and at high stroke risk. Follow up DSA was performed at 1-year. After that, image follow up was done by CTA, MRA or DSA. The patients were followed up at least 3 years.

Results 43 patients treated with Wingspan stent for symptomatic intracranial stenosis were enrolled in this study. The frequency for any stroke or death within 30-days was $7 \%$. The frequency of any stroke or death after 30-day was also $7 \%$ at mean 58.7 months follow up period. The frequency of $>50 \%$ restenosis on 3-year follow up image was $18 \%(n=8 / 43)$. There was complete occlusion in $9 \%(n=4 / 43)$. Reballooning was done in the other 4 patients but final occlusion was seen in 2 patients.

Conclusions The use of Wingspan stent in patient with $>50 \%$ symptomatic intracranial stenosis is associated with good long term clinical outcome. Long term restenosis rate was not high and asymptomatic in most patients. The incidence of symptomatic infarct was low among the restenosis patients.

Disclosures S. Park: None. S. Seo: None. J. Kim: None.

\section{E-109 NATIONAL TRENDS SHOW IMPROVEMENT OF CLINICAL OUTCOME WITH ISCHEMIC STROKE THROUGH ADVANCES IN ENDOVASCULAR THERAPY}

${ }^{1} \mathrm{~K}$ Seo*,${ }^{2} \mathrm{~S}$ Suh, ${ }^{3} \mathrm{~K}$ Lee, ${ }^{4} \mathrm{M}$ Kang. ${ }^{1}$ Neurology, National Health Insurance Service Ilsan Hospital, Goyang, Korea, Republic of; ${ }^{2}$ Radiology, Gangnam Severance Hospital, Yonsei University College of Medicine, Seoul, Korea, Republic of; ${ }^{3}$ Neurology, Gangnam Severance Hospital, Yonsei University College of Medicine, Seoul, Korea, Republic of; ${ }^{4}$ Institute of Health Insurance and Clinical Research, National Health Insurance Service Ilsan Hospital, Goyang, Korea, Republic of

10.1136/neurintsurg-2020-SNIS. 142
Background and Purpose Endovascular therapy (ET) of ischemic stroke with large vessel occlusion has been proved effective by clinical trials and reported with good outcomes in real clinical settings since 2015. We aim to determine national trends of clinical outcome in ET and compare the outcomes before and after mechanical thrombectomy.

Methods We obtained all data from the nationwide database of Health Insurance Review \& Assessment Service from 2008 to 2017. The patients with ischemic stroke who received ET were identified using International classification of disease-codes and several codes regarding to ET. In evaluating prognosis of patients receiving ET, good outcome was defined as discharge to home and poor outcomes as cerebral hemorrhage, physical disabilities and death. We analyzed the time points of ET to determine the factors affecting the prognosis. The study period was divided into three periods: 1.when stent-retriever was not used (non-ET period), 2.when it was used for off-label and non-reimbursement (transitional period), and 3.when it was reimbursed (ET period).

Results A total of 15,589 patients who were received ET between January 2008 and December 2017 were analyzed. Of the total subjects, 5,512 patients (35.4\%) received intravenous tPA. 3,028 were treated at non-ET period, 4,113 were treated at transitional period and 5,360 were treated at ET period. The rate of home discharge was $35.9 \%, 39.7 \%$ and $41.7 \%$ respectively, which was statistically significant $(\mathrm{p}<0.0001)$. 3-months mortality were $25.7 \%, 22.0 \%, 19.5 \%$ $(\mathrm{p}<0.0001)$, and 1-year mortality were $34.5 \%, 30.1 \%$ and $26.4 \%(\mathrm{p}<0.0001)$. There was a decrease in the likelihood of death (hazard ratio 0.693 , 95\% CI $0.645-0.744$ ) in ET period. The rate of disability was $33.6 \%, 26.5 \%$ and $23.4 \%$ $(\mathrm{p}<0.0001)$.

Conclusions The nationwide health insurance data showed clinical outcomes of patients with ischemic stroke after ET were significantly better in the ET period in Korea.

Disclosures K. Seo: None. S. Suh: None. K. Lee: None. M. Kang: None.

\section{E-110 PATIENT COUNSELING ON SMOKING CESSATION FOLLOWING ISCHEMIC STROKE}

${ }^{1} \mathrm{~K}$ Swafford*, 1J Frank, ${ }^{2} \mathrm{~J}$ Roberts, ${ }^{1} \mathrm{~A}$ Trout, ${ }^{3} \mathrm{~S}$ Grupke, ${ }^{4} \mathrm{~K}$ Pennypacker, ${ }^{3} \mathrm{~J}$ Fraser. ${ }^{1}$ Neurology, University of Kentucky, Lexington, $K Y ;{ }^{2}$ Neurosurgery, Neuroscience, University of Kentucky, Lexington, $K Y$; ${ }^{3}$ Neurosurgery, University of Kentucky, Lexington, $K Y_{\text {; }}$ ${ }^{4}$ Neurology, Neuroscience, University of Kentucky, Lexington, $K Y$

\subsection{6/neurintsurg-2020-SNIS.143}

Introduction/Purpose Ischemic stroke is the second leading cause of death in the United States. Smoking accelerates the onset of stroke, on average, by ten years. We sought to analyze the effects of smoking status following endovascular treatment (EVT) for large vessel occlusion in patients with acute ischemic stroke. Through our previously published Blood And Clot Thrombectomy Registry And Collaboration (BACTRAC) stroke tissue bank (ClinicalTrials.gov: NCT03153683), we aimed to determine the effects of smoking on the change in National Institutes of Health Stroke Scale (NIHSS) score, infarct volume, and cerebral edema volume. 
Materials and Methods Patients were divided into three groups: current smoker, previous smoker (defined as having quit greater than 6 months prior to the ischemic event), and non-smoker. NIHSS scores were recorded upon admission and at discharge. The change in NIHSS score was defined as score at admission minus the score at discharge. Infarct and cerebral edema volumes were measured. Linear regression analysis was performed based on infarct or edema volume versus the change in NIHSS score.

Results Eighty-eight adult subjects ( $>18$ years) were included in the study, of which 48 (55\%) were female. Median age was 69 years (25-101). Twenty-three (26\%) were active smokers, 14 (16\%) were previous smokers, and 51 (58\%) were non-smokers. Mean infarct time, or mean time from last known well, was $508 \pm 347$ minutes. Mean infarct volume was $67,882 \pm 90,632 \mathrm{~mm}^{3}$. Mean edema volume was $69,432 \pm 93,878 \mathrm{~mm}^{3}$. Mean NIHSS score on admission was $16.9 \pm 7.5$ and mean NIHSS score at discharge was $8.9 \pm$ 8.4. Mean age at time of infarct for the smoking population was 10 years earlier when compared to the nonsmoking population, 61.9 versus 71.6 years. Linear regression was significant $(\mathrm{p}<0.0001)$ when comparing infarct or edema volume versus change in NIHSS score for current smokers only.

Conclusion On average, smokers had stroke 10 years earlier than non-smokers, which is consistent with previously published findings. Smokers also had a statistically significant correlation between infarct or edema volume and worsening change in NIHSS score, when compared to both previous smokers and non-smokers who had no significant correlation. These findings are valuable in patient counseling for smoking cessation. Patients who continue to smoke are more likely to have a stroke earlier in life with potentially more significant disability and worse prognosis.

Disclosures K. Swafford: None. J. Frank: None. J. Roberts: None. A. Trout: None. S. Grupke: None. K. Pennypacker: 6; C; co-owner of Cerelux, LLC. J. Fraser: 2; C; Stream Biomedical, Medtronic, Penumbra. 4; C; Cerelux, LLC, Fawkes Biotechnology. 6; C; owner of Cerelux, LLC, owner of Fawkes Biotechnology.

\section{E-111 INTRACRANIAL CAROTID RUPTURE DURING THROMBECTOMY WITH EFFECTIVE TANDEM OCCLUSION}

${ }^{1} \mathrm{E}$ Cora*, ${ }^{2} \mathrm{~A}$ Arthur, ${ }^{1} \mathrm{M}$ Schmidt, ${ }^{1} \mathrm{R}$ Vandorpe, ${ }^{3} \mathrm{~B}$ Drake, ${ }^{4} \mathrm{D}$ lancu, ${ }^{3} \mathrm{H}$ Lesiuk, ${ }^{1} \mathrm{D}$ Volders, ${ }^{1} \mathrm{~T}$ Huynh. ${ }^{1}$ Department of Diagnostic Radiology, QEIl Health Sciences Centre, Halifax, NS, Canada; ${ }^{2}$ Semmes-Murphey Clinic, Memphis, TN; ${ }^{3}$ Department of Neurosurgery, The Ottawa Hospital, Ottawa, ON, Canada; ${ }^{4}$ Department of Diagnostic Radiology, The Ottawa Hospital, Ottawa, ON, Canada

\subsection{6/neurintsurg-2020-SNIS.144}

Introduction Mechanical thrombectomy has become the standard of care for patients presenting with acute ischemic stroke due to a large vessel occlusion. Intracranial hemorrhage is a serious complication which may occur during this procedure. The more commonly recognized cause of intraprocedural intracranial hemorrhage is due to iatrogenic vessel perforation. Here we present a series of patients with terminal ICA occlusions and proximal carotid stenosis that had mechanical thrombectomy complicated by devastating subarachnoid hemorrhage. Similarities between cases are highlighted and we hypothesize on the possible mechanism.

Case Description Three patients presented acutely with stroke symptoms due to an intracranial large vessel occlusion and had thrombectomy performed. Two of these patients had a tandem proximal and distal ICA occlusion extending to the M1 MCA with stenosis at the origin of the ICA. During the mechanical thrombectomy procedure all three patients had significant subarachnoid hemorrhage originating from the distal intracranial ICA in the region of the distal dural ring. The patient who did not have stenosis at the origin of the ICA, had the procedure performed with a balloon guiding catheter high in the cervical ICA which was relatively occlusive. Two patients had coiling performed to stop the hemorrhage, while the third patient was deemed for palliation. All three patients passed away within days after this serious complication.

Results We hypothesize that the cause of the subarachnoid hemorrhages in these patients was due to performing angiography in a closed system with the proximal stenosis/balloon guiding catheter causing blockage of the proximal ICA and the ICA terminus thrombus blocking the distal aspect. When performing an angiographic run, we hypothesize that the

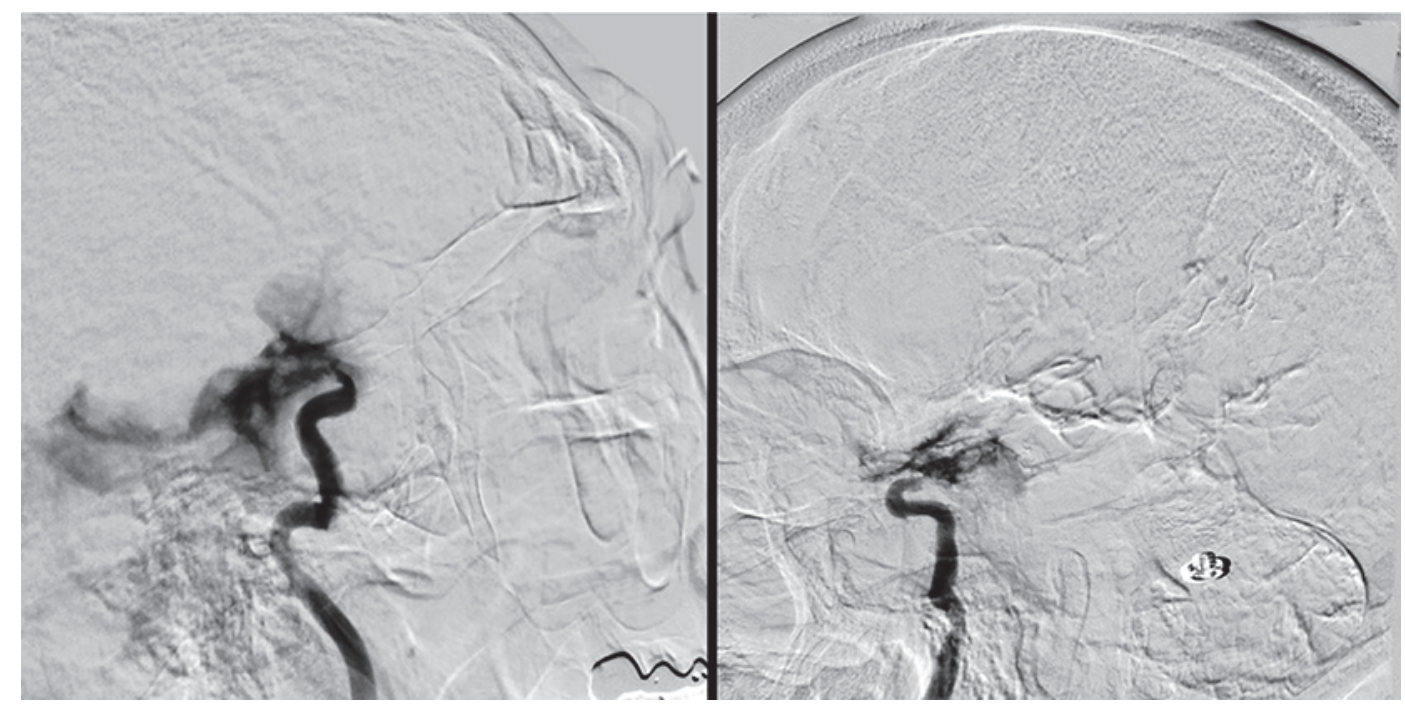

Abstract E-111 Figure 1 